https://helda.helsinki.fi

\title{
Working memory training mostly engages general-purpose large-scale networks for learning
}

\author{
Salmi, Juha
}

2018-10

Salmi , J , Nyberg , L \& Laine , M 2018 , ' Working memory training mostly engages general-purpose large-scale networks for learning ', Neuroscience \& Biobehavioral Reviews , vol. 93 , pp. 108-122 . https://doi.org/10.1016/j.neubiorev.2018.03.019

http://hdl.handle.net/10138/305544

https://doi.org/10.1016/j.neubiorev.2018.03.019

cc_by_nc_nd

acceptedVersion

Downloaded from Helda, University of Helsinki institutional repository.

This is an electronic reprint of the original article.

This reprint may differ from the original in pagination and typographic detail.

Please cite the original version. 


\section{Accepted Manuscript}

Title: Working memory training mostly engages general-purpose large-scale networks for learning

Authors: Juha Salmi, Lars Nyberg, Matti Laine

PII: $\quad$ S0149-7634(17)30717-0

DOI: $\quad$ https://doi.org/10.1016/j.neubiorev.2018.03.019

Reference: $\quad$ NBR 3077

To appear in:

Received date: $\quad 28-9-2017$

Revised date: $\quad 10-2-2018$

Accepted date: $\quad$ 17-3-2018

Please cite this article as: Salmi J, Nyberg L, Laine M, Working memory training mostly engages general-purpose large-scale networks for learning, Neuroscience and Biobehavioral Reviews (2010), https://doi.org/10.1016/j.neubiorev.2018.03.019

This is a PDF file of an unedited manuscript that has been accepted for publication. As a service to our customers we are providing this early version of the manuscript. The manuscript will undergo copyediting, typesetting, and review of the resulting proof before it is published in its final form. Please note that during the production process errors may be discovered which could affect the content, and all legal disclaimers that apply to the journal pertain. 


\section{Title}

Working memory training mostly engages general-purpose large-scale networks for learning

\section{Authors and affiliations}

Juha Salmi $^{1,2,3^{*}}$, Lars Nyberg ${ }^{4,5,6}$, Matti Laine ${ }^{1,7}$

${ }^{1}$ Department of Psychology, Åbo Akademi University, Turku, Finland

${ }^{2}$ Department of Psychology, Faculty of Medicine, University of Helsinki, Helsinki, Finland

3 Department of Psychology, University of Turku, Turku, Finland

${ }^{4}$ Department of Integrative Medical Biology, Umeå University, Umeå, Sweden

${ }^{5}$ Center for Functional Brain Imaging (UFBI), Umeå University, Umeå, Sweden

${ }^{6}$ Department of Radiation Sciences, Umeå University, Umeå, Sweden

${ }^{7}$ Turku Brain and Mind Center, University of Turku, Turku, Finland

\section{Corresponding author*}

Juha Salmi, PhD

E-mail: juha.salmitaival@abo.fi

Phone: +358405118678

Address: Department of Psychology, Åbo Akademi University, Fabriksgatan 2, 20500 Turku, Finland 


\title{
Highlights
}

- We performed the first meta-analysis of fMRI studies on working memory (WM) training

- We demonstrated that WM training modulates only the canonical WM network

- This general-purpose brain network was also modulated by perceptual-motor learning

- $\quad$ Only DLPFC/VLPFC was highlighted in WM training studies

Transfer to similar untrained tasks may be linked to prefrontal-striatal system

\begin{abstract}
The present meta-analytic study examined brain activation changes following working memory (WM) training, a form of cognitive training that has attracted considerable interest. Comparisons with perceptual-motor (PM) learning revealed that WM training engages domain-general largescale networks for learning encompassing the dorsal attention and salience networks, sensory areas, and striatum. Also the dynamics of the training-induced brain activation changes within these networks showed a high overlap between WM and PM training. The distinguishing feature for WM training was the consistent modulation of the dorso- and ventrolateral prefrontal cortex (DLPFC/VLPFC) activity. The strongest candidate for mediating transfer to similar untrained WM tasks was the frontostriatal system, showing higher striatal and VLPFC activations, and lower DLPFC activations after training. Modulation of transfer-related areas occurred mostly with longer training periods. Overall, our findings place WM training effects to a general perception-action cycle, where some modulations may depend on the specific cognitive demands of a training task.
\end{abstract}

Keywords: brain imaging, cognitive training, executive function, fMRI, plasticity, working memory 


\section{Introduction}

Human goal-directed behavior, including reasoning, planning, and decision-making, relies largely on our ability to maintain, manipulate, and update information contents in mind (Baddeley 1986). This ability, working memory (WM), not only provides a mental platform that can be used in pursuing our ongoing goals, but it is also a key precursor of long-term learning (e.g., Passolunghi 2007). The central role of WM in human cognition, coupled with its limited capacity, has motivated current attempts to improve WM function through intensive training (e.g., Constantinidis and Klingberg 2016). Over the last years, evidence accumulated in behavioral WM training studies has been meta-analyzed several times (Melby-Lervåg and Hulme 2013, Karbach and Verhaeghen 2014, Karr et al. 2014, Kelly et al. 2014, Shinaver et al. 2014, Au et al. 2015, Melby-Lervåg et al. 2016, Soveri et al. 2017). Some of these meta-analyses have found that WM training shows reliable transfer not only within the WM domain but also to attention and fluid intelligence (Shinaver et al. 2014, Au et al. 2015), while the most recent ones have documented substantial transfer only to untrained WM tasks (Melby-Lervåg et al. 2016), with only very weak effects in other cognitive domains (Soveri et al. 2017). Nevertheless, brain imaging studies that have accumulated only quite recently (see Table 1) have demonstrated multiple ways how WM training influences brain activity. These include regional changes in a multitude of areas (for a review see Buschkuehl et al. 2012, Constantinidis and Klingberg 2016, von Bastian and Oberauer 2014), modulation of anatomical (Takeuchi et al. 2010, Salminen et al. 2016, de Lange et al. 2017) and functional connectivity (Astle 
et al. 2015, Jolles et al. 2013, Takeuchi et al. 2013), adapted neuronal firing profiles (Meyers et al. 2012), and more efficient neurotransmitter function (Bäckman and Nyberg 2013). As there are limitations in the meta-analysis of behavioral studies, complementary analysis of the brain imaging studies could help in clarifying the mechanisms underlying WM training. For instance, more extensive transfer in some subset of studies could be of relevance but may disappear in behavioral meta-analyses when labeled together with different types of studies (for studies showing more transfer, see e.g. Holmes et al. 2009, Thorell et al. 2009, Brehmer et al. 2012, Bergman-Nutley and Klingberg 2014).

Several issues need to be considered when trying to relate the findings of the brain imaging studies on WM training to the broader context of training and skill learning. (1) Importantly, as these single studies have reported only the effects of WM training, it is not clear to what extent their findings are specific or shared with other types of learning tasks. WM emerges from a complex interplay between attentional processes, perceptual-motor (PM) systems, and long-term memory (see Eriksson et al. 2015 for a review), and thus WM training should share many fundamental elements with other types of skill training (see Chein and Schneider 2012). Nevertheless, WM training differs from training of other skills in that the typically adaptive WM training tasks force the participants to constantly work at their WM capacity limits, aiming to induce plasticity in the neural systems responsible for maintaining, manipulating and updating information. Hence, it would be important to clarify the specificity vs. generality of brain activation changes following WM training by making a comparison to PM learning. (2) Brain imaging studies on WM training have reported complex patterns of activation increases and decreases in large-scale brain networks that are difficult to interpret based on single studies. Relatively small samples and effect sizes, heterogeneous findings of individual studies (see Hsu et al. 2014), and the complex and distributed nature of the WM brain networks call for a search for replicable patterns across multiple studies. Systematic analysis of the existing neuroimaging findings could help in clarifying the role of 
specific brain areas in the widespread brain networks modulated by cognitive training. (3) Transfer of training to untrained tasks has been a key issue in behavioral WM training research. Even though there are still only few studies specifically examining the neuronal mechanisms underlying transfer, it is important to try to link the transfer-related brain imaging evidence to the behavioral transfer pattern. These three questions motivated us to conduct the first meta-analysis of the functional magnetic resonance imaging (fMRI) studies on WM training.

Brain networks involved in WM and PM tasks. NeuroSynth database includes about 900 published cross-sectional fMRI studies that have examined WM-related brain activations, making it one of the most studied neurocognitive systems (see Figure $2 b$ ). The brain regions consistently reported in these studies include those assumed to support WM core functions, namely the dorsal attention network (the superior parietal cortex/intraparietal sulcus, SPL/IPS; frontal eye field, FEF), dorsolateral prefrontal cortex (DLPFC), salience network (the supplementary motor area/anterior cingulate cortex, SMA/ACC, and inferior frontal gyrus/anterior insula, IFG/ant. ins.), as well as the fronto-striatal and cerebro-cerebellar loops connected with several of these areas (Figure 2b). Even though these brain networks show high consistency across single WM studies (see Owen et al. 2005, Nee et al. 2008), the large-scale activation patterns vary depending on specific WM component processes and content-specific perceptual representations exploited in a given task (see Eriksson et al. 2015). That is, when the WM task requires, for instance, operating with verbal, spatial or object-based information, the specific lower-level PM representations are also recruited (see Jonides and Smith 1999). Thus, in this theoretical framework, the WM component processes are essentially attention control processes (e.g., sustained attention, selective attention, inhibition, updating, Eriksson et al. 2015) acting on the relevant PM representations.

Of the extensive WM core regions listed above, DLPFC in particular is thought to be crucial for WM functions (Levy and Goldman-Rakic 2000, see also Figure 2b). Evidence spanning from single 
neuron studies (Goldman-Rakic 1996) to patient data (Muller and Knight 2006) and brain imaging findings (Owen et al. 2005, Eriksson et al. 2015) demonstrates that this area is related to distinct WM processes, especially in resilient information maintenance (Levy and Goldman-Rakic 2000), and parsing of related high-dimensional content associations (Miller and Cohen 2001). In addition to WM, DLPFC is also considered to contribute to other memory-related functions, for instance, recalling information with minimal retrieval cues or when minimal contextual information is available (Rugg and Vilberg 2013, see also Henson et al. 1999). As a hub of WM, DLPFC is heavily connected with all other WM-related brain regions that are described below in more detail.

The dorsal attention network and the salience network, together forming the so-called frontoparietal networks, are considered to be more multi-functional than DLPFC (see Figure 2b). The dorsal attention network is involved in directing and sustaining the focus of attention in selective task contents (Corbetta and Shulman 2002, see Scolari et al. 2015 for a recent review) that is crucial for maintaining information in WM, but also needed in almost any other task requiring goaldirected attention (Duncan 2010). Hence, in addition to WM functions (Owen et al. 2005, Nee et al. 2008), this network has an established role in spatial attention (Mesulam 1981, Corbetta and Shulman 2002, Silver and Kastner 2009), language processing (Hickok and Poeppel 2004, Saur et al. 2008), and motor functions (Rizzolatti et al. 2002, Culham and Valeyar 2006, van Polanen and Davare 2015). At the intersection between perceptual and motor functions, especially the parietal cortex is thought to integrate higher-level perceptual information with higher-level motor representations (Goodale and Milner 1992, see also Salmi et al. 2014). The salience network, in turn, contributes to the provision of fast access to the motor system together with facilitation of the detection of potentially important stimuli, and the regulation of switching between other large-scale brain networks when attention and WM resources are required (Menon and Uddin 2010).

Fronto-striatal and cerebro-cerebellar loops, traditionally considered to be mostly involved in motor 
function, have repeatedly been shown to be involved also in cognitive support functions such as anticipation, timing and response control, thereby contributing to optimization of cerebral function in diverse tasks (Marvel and Desmond 2010, Scimeca and Badre 2012). The functional roles of specific striatal and cerebellar areas are characterized by their anatomical projections (see Middleton and Strick 2000, Alexander et al. 1986). For instance, reciprocal connections between the neocerebellum (Crus I/II) and DLPFC can be utilized in WM functions, while tasks with high motor demands encompass the network connecting anterior cerebellum with the motor cortex (Salmi et al. 2010). There is some evidence that the dorsal striatum that is involved in regulating cognitive functions, may also have similar region-specific functional organization (see Pauli et al. 2016).

In sum, several brain areas in the so-called WM networks play diverse roles also in various other tasks operating with similar PM representations, even when those tasks do not load much on WM (see Figure 2b). This provides further impetus to the present attempt to identify general vs. specific features of brain activity changes following WM training. Possible unique features of WM training could relate to specific regions not seen in PM learning and/or to specific dynamics of change (activity increase or decrease) within a shared region. Domain-general changes in multi-functional brain networks, in turn, could reflect overlapping attention control functions involved in both WM and PM tasks.

Neuronal plasticity of WM and PM functions. While PM training studies provide some support for potentially lasting changes in brain anatomy (see Zatorre et al. 2012, Wenger et al. 2017), there is limited evidence of such effects in WM training research (see, however, Takeuchi et al. 2010, Salminen et al. 2016, de Lange et al. 2017). As training-related improvements in WM task performance typically decay relatively fast (Melby-Lervåg and Hulme 2013), also modulations of brain activity measured with functional brain imaging can be assumed to mostly reflect temporary 
flexibility in the neural resources dedicated to task performance, rather than plasticity observed as longer-term structural changes in brain tissue (Lövden et al. 2010, see also Buonomano \& Merzenich 1998).

In addition to WM, each brain network for goal-directed attention described above is also engaged in various other learning-related functions (Figure 2c, see also Chein and Schneider 2005, Chein and Schneider 2012). Concerning DLPFC, its role in learning is considered to depend on the amount of controlled processing required by the task (Blumenfeldt and Ranganath 2007). Heavy DLPFC engagement would thus be expected for learning tasks requiring constant access to existing or newly encoded task-relevant information, albeit these demands may decrease when learning progresses. By regulating the access to relevant information, DLPFC can promote learning of diverse functions related to both encoding and retrieval in declarative (Braver et al. 2001, Cabeza et al. 2002, Sakai 2003) as well as in procedural memory (Seidler et al. 2012). Also the fronto-striatal and cerebro-cerebellar loops have an established role in diverse learning functions ranging from action planning to decision-making and stimulus-response learning (Boyden et al. 2004, Liljeholm and O'Doherty 2012, Bäckman and Nyberg 2013). Activation changes in the frontoparietal networks are likewise consistently reported in different types of learning-related studies, although the exact role of these areas in learning is less clear (see Chein and Schneider 2005 for a metaanalysis). What has been reported in several cross-sectional studies is that the activity in distinct areas of these networks shows specific links to individual task performance. While in several prefrontal areas higher activity predicts improved task performance, in the parietal cortex lower activations have been associated with higher task performance (e.g., Braver et al. 1997, Honey et al. 2000, see also Pessoa et al. 2002, Linden et al. 2003). That is, both increases and decreases in brain activity have been found to be associated with higher WM capacity, and this may vary in different brain regions. 
Learning is often characterized as a changing interplay between controlled and automatic processes (Shiffrin and Schneider 1977). Controlled processing is typically associated with attention and active monitoring of the task performance, and it is considered to play an important role in unfamiliar tasks especially when they are cognitively demanding. Automatic processing, in turn, emerges or strengthens during practice, saving the limited resources for controlled processing that are critical in the development of expertise. It has been suggested that changes in controlled and automatic processing are related to increases and decreases of brain activity, respectively (e.g., Chein and Schneider 2012). Both types of activation changes have been widely reported in WMrelated regions pre vs. post WM training (see Buschkuehl et al. 2012, and von Bastian and Oberauer 2014, for reviews). This is also the case for PM learning involving similar representational systems as in WM training but with a minimal WM load (Chein and Schneider 2005). In theory, increased activity could reflect a higher number of neurons engaged or higher firing rates (Qi et al. 2011, Meyer et al. 2011). Decreased activity, in turn, could reflect higher efficiency, that could be mediated by increased specificity to detect stimuli (narrower tuning curves, Raine and Miller 2000), or a lower number of neurons required to perform the task. However, as noted in a recent review (Constantinidis and Klingberg 2016), increases and decreases of blood oxygenation dependent signal are only weakly linked to the underlying neurobiological mechanisms. Such mechanisms that are beyond the range of functional brain imaging include, for example, altered gene expression, structural changes in axons and dendrites, as well as changes in spiking mechanisms not influencing total energy consumption (see Holtmaat and Svoboda 2009). In addition to very complex underlying brain functions, also the links between the task performance, cognitive load, and brain activations are complex, and may be varying not only in different brain regions but also depending, for instance, on the age of the participants (Schneider-Garces et al. 2010).

Despite complex underlying mechanisms, analysis of the similarities and differences between training-related changes induced by WM and PM training could potentially provide important 
information about the domain-specificity vs. domain-generality of these learning processes. Especially the early studies focusing on PM learning strongly emphasized decreased activations, possibly indicating higher automaticity in the widespread cognitive control networks as well as in areas involved in lower-level PM functions (Chein and Schneider 2005). At the time of the first meta-analysis on this topic by Chein and Schneider (2005) there was, however, not enough data to distinguish between different types of learning tasks and the sample sizes in individual studies were very small (mean sample size 10 participants in the 25 studies included). Brain imaging studies on WM training, which appeared later, have highlighted enhanced activity in the dorsal attention network and striatum (Olesen et al. 2004, for a review see Constantinidis and Klingberg 2016). At that point, the existing evidence suggested that training PM vs. WM tasks might largely show distinct neuronal effects. As indicated above, later studies have changed this early view. First, there are now a number of studies reporting activation increases in the dorsal attention network and salience network even during simple PM learning tasks (see Figures 3 and 4 for these effects). Second, an accumulating number of WM training studies have reported activity increases or decreases in areas primarily involved in lower-level sensory-motor functions (see Table 1 for a comprehensive list of brain imaging studies on WM training). Given that the direction of activation changes as well as areas showing training-related modulations have varied considerably in individual studies (see Supplementary Figure 1), we hoped that our systematic analysis of the fMRI findings in WM and PM training studies would allow interpreting WM training effects in the more general context of learning-related brain plasticity.

\section{Incorporating functional brain imaging studies into the broader context of WM training}

research. Cognitive training represents an extensive multidisciplinary research domain that covers a wide range of target functions and populations. Training can be based on a single task or multiple tasks (Cheng et al. 2012, Binder et al. 2016), seeking improvements by performing tasks at maximal load or by learning new strategies to perform difficult tasks (Turley-Ames and Whitfield 2003, St 
Clair and Thompson 2008), and the training outcomes can be measured in various ways ranging from cognitive tasks to subjective ratings, symptom measures, and daily activities. In addition to training regimes simply focusing on repetitive performance of the same tasks, some studies have examined joint effects of cognitive training and conventional academic tasks (Nemmi et al. 2016), aimed for improvement of everyday attention control functions (see Lilienthal et al. 2013), or examined the effects of concomitant brain stimulation (Richmond et al. 2014, Nilsson et al. 2017, Ruf et al. 2017, Stephens et al. 2017). Brain imaging evidence is still lacking for many of these approaches developed in behavioral research.

One of the most carefully examined research questions in behavioral WM training research that has also been addressed in some brain imaging studies is whether the training effects carry over from one task to another. Behavioral transfer in individual WM training studies has varied considerably between studies: some have found no transfer, others near transfer to untrained WM tasks, or far transfer to other executive functions and even fluid intelligence (Hsu et al. 2014). Recent metaanalyses suggest that besides consistent improvements in the trained tasks (practice effect), the only more substantial transfer following WM training concerns other, untrained WM tasks (e.g., MelbyLervåg et al. 2016), mostly structurally similar task variants (Soveri et al. 2017). Thus, similarly to PM learning, WM training fails to provide widespread transfer. It should be noted, however, that a meta-analysis may not detect all the relevant effects due to the heterogeneity of the individual studies. Some studies have suggested that effects are more consistent when specific training approaches are utilized (e.g., Shinaver et al. 2014).

While only a limited number of brain imaging studies have addressed transfer after WM training, they can provide complementary evidence on WM training mechanisms. Potentially strongest evidence for altered neurocognitive function would come from findings pointing to recruitment of new brain networks that were not activated prior to training (see Buschkuehl et al. 2012, von 
Bastian and Oberauer 2014). This could be the case, for instance, if WM training influences the executive system (Karbach and Verhaeghen 2014, Karr et al. 2014, Au et al. 2015). Another account suggests that the more similar the activity between the trained and untrained tasks, the more likely it is to observe transfer (Dahlin et al. 2008). Hence, this approach suggests that transfer is mostly observed in similar tasks and within the same brain networks. It has been suggested that such transfer within the same brain networks could manifest as a shift from prefrontal controlled functions to more automatized striatal functions (Dahlin et al. 2008, Kuhn et al. al 2013, Salminen et al. 2016). Due to the high cognitive and neural overlap between attention and WM, transfer could also be mediated by the networks for goal-directed attention. There are thus several proposals on the neurocognitive mechanisms of WM transfer, and it is important to analyze the available neuroimaging evidence to evaluate them.

Aims and goals of the study. The first goal of this study was to advance our understanding on the nature of WM training effects. Although early studies reporting short-term practice effects demonstrated that exposure to a WM task changes the organization of the brain networks by recruiting new functional systems (Kelly et al. 2006, see also Buschkuehl et al. 2012), accumulating evidence from longer-term WM training studies suggests that neuronal plasticity manifests largely within the canonical WM networks (Constantinidis and Klingberg 2016). We attempted to verify this by compiling and analyzing all related empirical research evidence. As a reference for taskspecific training effects, we obtained several large-scale meta-analyses from Neurosynth database for studies reporting cross-sectional (non-training) activations for similar tasks (Figure 1 and Figure 2). The second goal was to analyze the available fMRI evidence by meta-analytic means to delineate the brain networks involved in WM training, and to examine whether these areas are similar to those involved in learning PM tasks in which WM load is minimal (Figure 1 and Figure 3). Accordingly, we collected data from WM training studies and compared these data to findings of prior meta-analyses on PM training (Chein and Schneider 2005, Davis and Gaskell 2009, 
Hardwick et al. 2013). As PM training studies typically have shorter training periods than WM training studies, we separately analyzed WM training effects for studies with shorter (less than two weeks) and longer (more than two weeks) training period (Figure 5, see also Table 1). We also separated between WM training studies utilizing verbal and visuospatial tasks in order to compare WM and PM studies that engage similar stimulus representations (Figure 4). Thirdly, we separated between training-induced activation increases and decreases to make inferences on the changes in automatic vs. controlled processing in WM and PM training (Figure 1 and Figure 6). Similar training-related dynamics of WM and PM training would speak for an all-purpose learning network for goal-directed tasks. WM-specific effects, in turn, could provide support to the original idea with WM training, i.e., that such training could specifically enhance functioning of WM core systems (see Klingberg 2010). Finally, we segregated between the effects reported for trained WM tasks and untrained WM transfer tasks to reveal whether there are brain systems consistently reported across studies that underlie transfer effects (Figure 1 and Figure 7). As transfer effects in behavioral studies have mostly been quite modest, we expected that also activation changes in untrained transfer tasks would be rather limited. Even considering the narrow transfer effects, the loci of training-induced activation changes in untrained tasks could provide important information on the mechanisms involved in transfer. For instance, these results could speak for the role of prefrontal executive systems, large-scale networks for goal-directed attention, or shift of balance from controlled prefrontal functions to more automatized striatal functions underlying transfer.

\section{Materials and Methods}

Baseline activation maps. We used automated term-based meta-analyses in Neurosynth to obtain reference maps for the training-induced modulations in WM, language, spatial, and motor tasks (Figure 2b, see www.neurosynth.org/analyses/terms/). The meta-analytical forward-inference maps were obtained in June 2017. These meta-analyses included in total 901 WM studies, 885 language 
studies, 1157 spatial studies, and 2081 motor studies. It was confirmed that the regional overlap between WM studies and PM studies was relatively low (1-4\%). Note that these term-based metaanalyses were not performed to establish an accurate comparison of cross-sectional WM, language, spatial, and motor studies, but to obtain approximate maps for peak activations in a sample with maximal coverage.

Meta-analysis study selection. Keywords "working memory", "executive function", "fMRI", and "training" produced a vast amount of potentially eligible studies: PubMed 311 hits; MedLine 136 hits; PsycInfo 115 hits; Google Scholar 45800 hits of which only 69 had WM, training, and fMRI or brain in the title. The numbers are from the original search conducted in February 2016. A revised search resulting in 3 new eligible studies was conducted in June 2017. In order to reduce the heterogeneity of the data, we excluded studies with children and clinical groups. From the studies found with the keywords above, we excluded those that were not conducted with fMRI and/or did not contain training interventions, did not describe the training task clearly (e.g., commercial cognitive training softwares tapping several cognitive functions), included less than five participants, or did not report standard-space coordinates. Authors of seven potentially eligible studies not reporting coordinate data were contacted by e-mail, but no unpublished data that would have allowed inclusion of some of these studies was obtained. An additional search was conducted in order to double-check other studies of the first and last authors of the studies included on the first pass. Finally, we went through all individual studies cited in several key review papers on this topic in order to confirm the coverage of our sample. All in all, 26 studies with 316 foci were included in the present analysis (see Table 1).

PM studies were selected based on previous meta-analyses on this topic. More specifically, Chein and Schneider (2005) included both sensory-motor and perceptual learning studies on their metaanalysis which were divided to those training spatial or verbal/object tasks, or lower-level motor 
functions. Davis and Gaskell (2009) did a meta-analysis focusing on verbal learning, providing us several more potential verbal studies. Finally, Hardwick et al. (2013) meta-analyzed PM studies either focusing on learning of simple motor sequences or perceptual learning mostly involving spatial functions. In order to provide strict experimental control, we selected only those studies which reported learning effects isolated as a contrast between the brain activation prior to vs. post training phase. Our final data pool included 20 motor (370 foci, 284 participants), 15 spatial (284 foci, 175 participants), and 18 verbal (259 foci, 223 participants) studies. For each study, we separately coded the activity increases and decreases.

The sample included 439 foci in 50 studies reporting activity increases. Of these foci, 124 were from WM studies, 98 from spatial studies, 129 from motor studies and 98 from verbal studies. 568 foci in 53 studies concerned activity decreases. Of these foci, 89 were from WM studies, 137 from spatial studies, 235 from motor studies and 136 from verbal studies. Furthermore, for the WM training studies we separated between trained (criterion task in fMRI) vs. untrained tasks (transfer task in fMRI). Activation during the trained criterion task was examined in 20 studies (259 foci), and activation in an untrained transfer task was reported in 8 studies (30 foci). Of these activations, in trained tasks 110 foci in 13 experiments were activation increases and 77 foci in 9 studies were activation decreases. In untrained transfer tasks, 19 foci in 4 experiments were activation increases and 33 foci in 5 experiments were activation decreases. For WM training studies, names of the first authors, sample sizes (training group), inclusion and type of control group, analysis of transfer effect, training approach, activity increases vs. decreases reported, and durations of the training period are shown in Table 1. PM training studies are listed in Supplementary Table 1. Supplementary Table 2 describes the behavioral effects reported in WM training studies.

Activation likelihood estimation. GingerALE software (version 2.3.6, www.brainmap.org) was used to generate activation likelihood estimation (ALE) based convergence maps in different 
conditions (Eickhoff et al. 2009, Turkeltaub et al. 2012). This software models coordinate data as spatially smoothed 3D Gaussian probability distributions capturing the uncertainty associated with each locus. The smoothing kernel (full-width half maximum) is determined by the number of subjects included in the experiment (e.g., 1 participant $=19.1 \mathrm{~mm}, 5$ participants $=11.4 \mathrm{~mm}, 10$ participants $=10 \mathrm{~mm}, 50$ participants $=8.75 \mathrm{~mm}$ ). Hence, narrower filter width is associated with larger samples having greater spatial certainty (Eickhoff et al. 2009). GingerALE uses a separate algorithm to join the probabilities of nearby foci to avoid overweighting experiments that report cluster subpeaks (Turkeltaub et al. 2012).

ALE values were calculated by the voxel-wise union of the probabilities in the modeled activation maps. ALE maps were then thresholded at corrected $p<0.05$ (cluster level $\mathrm{p}<0.05$, clusters defined through 100 permutations). Separate ALE maps were computed for all training effects (post- vs. pre-training activity), contrasts between WM and PM effects (each contrast was run separately), activation increases and decreases for WM and PM conditions, and trained (training effect) and untrained (transfer effect) tasks. Studies reporting interaction effects were omitted from the analyses separately reporting activity increases vs. decreases. To match the PM and WM studies in terms of perceptual representations, we performed separate analyses for the spatial (99 foci in 10 experiments) and verbal (129 foci in 10 experiments) WM training studies that are the main categories of WM training studies. As PM training studies have typically used shorter training periods, the influence of training duration (see Table 1) in WM studies was further examined by separately analyzing the studies with training period shorter than two weeks (136 foci in 11 experiments) or longer than two weeks (180 foci in 15 experiments). Due to the relatively low number of studies, direct contrast between the two maps resulting from the analyses concerning training length and stimulus materials were not performed. To clarify the role of training duration and task type on the WM training, we performed separate analyses for these effects. The influence of training duration (see Table 1) was examined by separately analyzing the studies with training 
period shorter than two weeks (136 foci in 11 experiments) or longer than two weeks (180 foci in 15 experiments).

\section{Results}

Non-training effects. Figure $2 \mathrm{~b}$ illustrates the regional activity reported across WM, language, spatial, and motor brain imaging studies obtained from NeuroSynth database, providing reference maps for the training effects. This analysis revealed a large-scale brain network consisting of the frontoparietal and salience network, occipital and temporal cortical areas, and dorsal striatum that is overlapping across all term-based meta-analyses. Separate analyses for WM and PM studies showed clear differences only in DLPFC that was more consistently activated across WM studies than PM studies. Language studies encompassed more widespread temporal cortical and inferior prefrontal areas than other studies. Spatial studies specifically involved posterior parietal cortex (PPC) and occipital areas in the dorsal visual stream, as well as parahippocampal and hippocampal areas. For the motor studies, distinct activity was observed in lower-level motor areas, as well as in the putamen, and anterior cerebellum.

Training effects - Overall analysis. We performed detailed ALE meta-analyses for learning effects reported in WM and PM studies to determine their regional specificity vs. overlap (Figure 3). Overall, WM training studies have reported activations in each WM-related area (see Figure 2), and also in several other areas (Supplementary Figure 1). With the exception of the cerebellum, areas within each core WM systems also showed statistically significant training-related modulations. More specifically, analysis lumping together all WM training-related foci revealed consistent activations in several prefrontal areas (SMA/ACC, DLPFC, IFG, and FEF), PPC, and striatum (Figure 3a). Comparisons to PM learning studies revealed that several WM-related areas showing training effects are not selective to WM training (Figure 3a/b). Verbal learning tasks modulated 
activity in overlapping prefrontal areas, excluding SMA/ACC. As expected, these effects were more clearly observed in the left hemisphere. Learning effects on the motor tasks were observed in the striatum, cerebellum, thalamus, motor cortex, SMA, and FEF. Practice with spatial tasks influenced mostly activity in DLPFC and FEF. Contrast analysis confirmed that in DLPFC and VLPFC, WM training effects were larger than PM training effects (Figure 3b). This analysis also showed the important role of the ventral and dorsal occipital cortex and temporal cortex in verbal learning, the contribution of the striatum, thalamus, and cerebellum in motor learning, and the involvement of DLPFC in spatial learning.

Training effects - Spatial and verbal WM training. To match the stimulus representations between PM training and WM training studies, we separately analyzed WM training studies utilizing visuospatial and verbal tasks. Both types of training have been shown to modulate widespread networks in individual studies (Supplementary Figure 1b). The most consistent training-related modulations across spatial tasks are observed in the right DLPFC (Figure 4). In verbal tasks, training-induced changes are observed in more widespread brain networks consisting of both the dorsal attention network and the salience network, including the Broca's area in the left inferior frontal region (Figure 4).

Training effects - Short and long WM training period. Due to the high variability in the duration of the training period, we separately analyzed studies with less than two weeks or more than two weeks of training (Figure 5, see also Supplementary Figure 1c). Already studies with short training period have consistently reported activations in the salience network and in the dorsal attention network, and similar modulations in these two networks are consistently seen also in the longerlasting training studies. Unlike studies with shorter training period, longer training resulted also in modulations of the dorsolateral prefrontal and striatal activity. 
Training effects - Activation increases and decreases. In subsequent analyses, training-related activations were divided into increases and decreases (Figure 6, see also Figure 7b/c). Trainingrelated increases and decreases have been reported in multiple brain areas, but many of these are not showing consistent effects. This is the case especially with training-related decreases. Even though 568 foci in widely distributed areas have indicated activation decreases in 53 training studies, across all studies there was only one brain area, namely DLPFC, consistently showing activation decreases at cluster corrected $p<0.05$ threshold (Figure 6a). Training-related increases have been reported less frequently (439 foci in 50 studies), however, more consistently in areas overlapping across studies, namely the salience network, dorsal attention network, striatum, thalamus, ventral and dorsal visual, and superior temporal cortices (Figure 6a).

For domain-specific increases and decreases, we separately analyzed these effects in WM and PM studies. This analysis suggested that in WM studies, consistent activation increases have been reported in the salience network, anterior PFC, and striatum (Figure 6b). Decreased activity, in turn, has been observed in the visual and posterior parietal cortices (Figure 6b). Increased activity in the salience network (SMA/ACC specifically) and decreased activity in the sensory (both auditory and visual) and parietal areas was also observed in studies focusing on verbal learning (Figure 6c). Visual areas and some of the parietal areas showed consistent activity decreases also for spatial training effects (Figure 6d). However, in some of the midline parietal areas and SMA/ACC, the direction of the activity change appeared to be opposite to WM-training studies and additional modulations in the anterior cerebellum (increased activity) and posterior cerebellum (decreased activity) were observed (Figure 6d). Motor studies have reported increases and decreases of brain activity in mostly different areas than WM training studies, excluding the striatum (Figure 6e). In these studies, consistent activation decreases have been reported in DLPFC and thalamus in the vicinity of the pulvinar nucleus, and activation increases in the medial areas of the primary motor cortex (Figure 6e). 
Transfer effects - Trained and untrained tasks. Training effects in trained and untrained tasks have been reported in multiple WM-related areas. The areas specifically modulated in trained tasks include the salience network, dorsal attention network, and striatum (Figure 7a). In the untrained transfer tasks, significant activation across all studies was observed only in DLPFC (Figure 7a). However, in a more detailed analysis separating between activation increases and decreases in the trained and untrained tasks, the activation pattern was slightly different (Figure $7 \mathrm{~b}, \mathrm{c}$ ). As earlier analysis including all WM training-related activations suggested (see Figure 6b), in trained tasks activation decreases were observed in the parietal and visual areas, and activation increases were observed in the prefrontal cortex, and also in the striatum (Figure 7b). In turn, in the untrained transfer tasks, this analysis separating between activation increases and decreases showed higher striatal and IFG activation and lower DLPFC activation after training (Figure 7c).

\section{Discussion}

We set out to perform the first systematic analysis of fMRI studies on task-related brain activation changes following WM training. The goal of our study was to address four main questions: (1) Are training-induced activation changes limited to the canonical WM network or are there new areas involved after the training period? (2) To what extent the activation changes related to WM training overlap with those reported in learning of PM tasks? (3) Which brain networks manifest changes in automatic vs. controlled processes in WM vs. PM training? (4) Which of the observed brain activation changes following WM training reflect transfer to untrained tasks? We discuss the results concerning each of these main questions in a separate section (see Figure 8 for summary).

Are activation changes induced by WM training observed only within the canonical WM network? Comparison of the term-based analyses for cross-sectional (non-training) WM effects 
(Figure 2 yellow and white) and search-based meta-analysis for WM training effects (Figure 3b yellow) corroborated the recent suggestion by Constantinidis and Klingberg (2016) that training modulates brain activity mostly within the canonical WM networks (Figure 3b/c, see also Owen et al. 2005, Rottschy et al. 2012). Hence, training manifests as a redistribution of brain activity within the existing core networks, rather than as a reorganization with recruitment of additional neuronal resources from other large-scale brain networks (see also Buschkuehl et al. 2012, von Bastian and Oberauer 2014). These findings are broadly in line with the suggested neurobiological mechanisms associated with WM training (Constantinidis \& Klingberg 2016). That is, training-induced changes in the number of activated neurons, firing rates, and/or tuning curves can be assumed to take place within the localized brain systems that were engaged in task performance from the outset. These potentially rapidly occurring and then dissipating effects provide evidence of short-term plasticity related to functional activity (Buonomano \& Merzenich 1998). However, current brain imaging studies provide very little evidence of more permanent changes following WM training (Lövden et al. 2010, see however, Salminen et al. 2016, de Lange et al. 2017 for studies demonstrating structural changes).

WM training-related changes in brain activity have been reported basically in all main WM networks (Supplementary Figure 1). Of these networks, however, only modulations of the dorsal attention and salience networks, DLPFC, as well as striatum were statistically significant in metaanalytical observation. Based on these findings, it appears that the more important the role of the area is in WM, the higher the likelihood of training-related modulation (see e.g, Owen et al. 2005, Rottschy et al. 2012). Hence, current brain imaging evidence does not provide evidence of areas that would be sensitive to learning per se but rather emphasizes the modulation of the core systems. It could also be that potential changes outside the core systems are not observable here because of multiple factors that are likely to influence the pre-post activation changes in various ways in different studies. The amount of studies was marginally sufficient to examine the mediating effect 
of training duration. This analysis suggested that the activity in the salience network and dorsal attention network is modulated already after relatively short period of training, while striatal and DLPFC modulations appear to be robust only after longer training (Figure 5). Another mediator that was shown to be associated with specific areas being activated was stimulus materials. Separate analyses for verbal and spatial WM training studies suggested consistent modulations of the dorsal attention network and salience network, and DLPFC, respectively (Figure 4). It should be noted here that also many other areas have been observed to be modulated in verbal and spatial WM training studies, but from this relatively modest subsample, these areas showed most robust activations (see Supplementary Figure 1b). Other factors that could play a role here include participants' strategy use, load of the trained task, level of performance and training-related changes in performance features, and the type of the training task and the pre-post task. Currently, the available data does not, however, allow conducting a reliable analysis that would account for these effects. Nevertheless, as these factors are directly related to WM performance, they can also be expected to result to training-related modulations mostly within the core WM networks.

\section{Are the activation changes related to WM training similar to those reported in learning of PM}

tasks? The analyses of non-training studies showed that canonical WM networks are activated not only by WM tasks but also by PM tasks relying on overlapping attention control functions, supporting the current view that no cognitive-neural processes are totally unique to WM (Eriksson et al. 2015; see Figure 2b). Both non-training as well as training studies suggested a special role for DLPFC in WM, albeit also PM studies, especially those operating with spatial and motor contents, showed training-related modulation of DLPFC function (Figure 3b, 4e). Nevertheless, as predicted, our results indicate that DLPFC is the WM hub (e.g., Levy and Goldman-Rakic 2000, Kane and Engle 2002, Curtis and D'Esposito 2003, Barbey et al. 2013) also in the case of WM training (Figure 3). The special role of DLPFC in WM training is also supported by studies in non-human primates showing that while the tuning of the neurons in this area to selective WM contents is 
increased during the training period, functioning of the cells that are activated during the presentation of stimuli does not show major changes (Meyer et al. 2011, Qi et al. 2011).

Interestingly, in an unfamiliar WM task, higher DLPFC activity has been shown to correlate positively with accuracy (e.g., Braver et al. 1997), but with extended training this activity appears to decrease (also suggested in Dahlin et al. 2008). In accordance with domain-general theories of learning (Chein and Schneider 2012), this would suggest that an unfamiliar task requires high attentional control, while during training performance becomes more routine and less dependent on these executive resources. The existing body of brain imaging literature indeed shows more consistent DLPFC activation decreases in motor studies than in WM studies. This could be due to steeper and less variable learning curves in motor tasks, with DLPFC likely being required only in the very beginning of training (see Chein and Schneider 2012). The existing pool of data suggests both increases and decreases of DLPFC activity in WM studies (this is why DLPFC does not appear as clearly in the analysis separating between activation increases and decreases as in the analysis of the overall training effects). However, due to the lack of relevant studies, heterogeneity of study designs, and other sources of variability, there is not enough evidence to reliably distinguish the influence of training duration and the slope of the learning curve on modulation of DLPFC activity (see Kuhn et al. 2013). It thus remains open whether the role of DLPFC in WM training that was highlighted in the present findings is more closely related to WM processes (e.g., initial requirements for attentional control in unfamiliar tasks that involve a high WM load) or to the general dynamics of learning in cognitively challenging tasks. Our analysis separating between shorter and longer WM training studies suggested that consistent DLPFC modulations are observed only across studies with training period lasting at least two weeks (Figure 7), while in PM training studies these areas were modulated even though the training period was clearly shorter. It could therefore be that DLPFC plays a differential role in WM and motor training. 
Also VLPFC was more consistently modulated in WM than in PM training studies (Figure 3b/c). In keeping with our findings, invasive electrophysiological recordings in non-human primates demonstrate that the number of VLPFC neurons activated specifically during WM performance is considerably increased during training (Meyer et al. 2011, Qi et al. 2011). However, due to the domain-general role of this region, at least three different alternatives are worth noting here: the direct contribution of IFG on WM (Crottaz-Herbette et al. 2004, Goulden et al. 2014, Manelis and Reder 2014) and memory encoding (Badre \& Wagner 2007), its role in language processing (see Liakakis et al. 2011), and its contribution to processing the relative importance of the incoming stimuli (Menon and Uddin 2010). The WM interpretation could be supported by the fact that our results contrasting WM and PM training revealed not only the left VLPFC (inherently involved in language processing) but also the right VLPFC involvement in WM training. Therefore, this issue requires further examination. When the amount of data on verbal vs. spatial WM training effects accumulates, this issue could possibly be solved with the means of a similar meta-analysis (see Badre \& Wagner 2007 for the role of right VLPFC in spatial memory). Neither can we rule out the possibility that training-induced modulation of VLPFC activity reflects more efficient selection of the relevant stimulus material (Menon and Uddin 2010).

The dorsal attention and salience networks were modulated by both WM and PM training, even though some differences were observed in the locations of the activation peaks within these networks (Figure 3). In the case of WM training, modulation of the dorsal attention network and salience network was observed already after relatively short training. Also PM training studies typically have relatively short training period. Hence, these findings together suggest that trainingrelated modulation in these areas is observed already after short training. Consistent activation increases were observed in areas close to FEF and premotor cortex in WM training, but not in PM training. Nevertheless, in the light of the present meta-analytic data, modulation of the dorsal attention network does not stand out against PM training studies. The most obvious reason for 


\section{5}

overlapping changes of activity in these networks is the similarly important role of attention in these two modes of training. In PM tasks the role of attention is relatively straightforward, as active processing of the incoming stimulus streams requires, for instance, sustained attention, directing of attention, and detection of salience of the incoming stimuli. Also the contemporary models of WM suggest that neither maintaining nor manipulating information in mind involve specialized brain networks, but these functions rather take place in brain networks also contributing to basic processes of goal-directed attention (Eriksson et al. 2015). As activation changes in these networks are proportional to improvement of WM task performance (Olesen et al. 2004, Beatty et al. 2015, Garner \& Dux 2015, Salminen et al. 2016), these networks appear to significantly contribute to the process of learning cognitive tasks.

Due to their domain-generality, modulations of the frontoparietal networks could potentially have even more widespread effects via their general role in attention control. Although there is extensive evidence of the role of these networks in WM training, including also enhanced functional connectivity (Jolles et al. 2013, Langer et al. 2013, Astle et al. 2015) and strengthened anatomical connectivity (Takeuchi et al. 2010, de Lange et al. 2017) as response to WM training, the role of these modulations of brain activity is difficult to interpret as it is still unclear whether there are behavioral effects that are coupled with these changes in brain activity (see, e.g., Melby-Lervåg et al. 2016, Soveri et al. 2017 for meta-analyses suggesting transfer limited within the WM domain versus Shinaver et al. 2014 and Au et al. 2015 suggesting broader transfer beyond the domain of WM). Our findings of similar activation and deactivation patterns in these areas as response to WM and PM training, would seem to limit the role of these networks in training to flexibility that is specific to the trained tasks. Although there could be a mediating role of attention in WM training, it might not be specific to WM training. There are some studies reporting WM training transfer to attentional functions (Chein \& Morrison 2010, Brehmer et al. 2012), but more empirical evidence is still needed to clarify this issue. 
The current WM training research has largely focused on premises of the capacity-based multidomain system. Recently, this view has been challenged (e.g., Macken et al. 2015) and the evidence placing WM into a more general perception-action cycle has accumulated also in the neuroimaging research (e.g., Eriksson et al. 2015). In this context, in future it would perhaps be more useful to examine in greater detail whether cognitive training could influence some of the core attention-related or PM functions, rather than to focus on possible trans-domain influences between higher-level executive functions that are shown to be weak, yet still statistically significant (Soveri et al. 2017).

The fronto-striatal loop, being a key system at the junction of the prefrontal cognitive control systems and motor circuitries, has been suggested to contribute to WM training in several studies (see Bäckman and Nyberg 2013 for a review). Our analysis provides further evidence on changes in striatal activity patterns associated with training (Figures 3, 4, and 5). As originally suggested by Dahlin et al. (2008), striatal activation increase parallels DLPFC activation decrease, possibly indexing a shift from controlled to more automatic processing. Despite the important role of striatum in WM updating (Bäckman and Nyberg 2013), we did not, however, find clear evidence for a special role of the striatum in WM training as compared to PM training and motor training. The only difference was that, apparently in case of WM training, modulation of this network occurs mostly after some weeks of training. Being the main input to the dorsal striatum (Alexander et al. 1986), PFC (and in the case of WM, mostly DLPFC) is the region that has a special role in modulating striatal functioning. It should be noted, however, that both DLPFC as well as the striatum are also connected to the frontoparietal networks (see, e.g., Choi et al. 2017). A further look at the possible specificity of the striatum in motor vs. cognitive training would benefit from a detailed analysis separating the anatomical pathways targeting specific striatal nuclei and resolving the role of specific prefrontal input areas in learning. 
Other WM-related networks, such as the visual cortex and cerebellum, showed more consistent activations in PM training than in WM training studies (see Figures 3, 6, and Supplementary Figure 1). In other words, even though modulations of these areas are widely reported in WM training studies, it could be that these modulations reflect changes in basic representational and motor functions that are shared between various tasks and play even a more important role in PM tasks than in WM tasks.

Which brain networks manifest changes in automatic vs. controlled processes in WM vs. PM training? Activation increases and decreases related to WM training showed similarities to the overall learning effects in PM studies, providing further evidence that WM training effects can be placed into a more general perception-action cycle (Nyberg and Eriksson 2015). Similar to verbal and spatial learning, WM training was associated with activation decreases in the visual and parietal cortices, and in line with motor training, it also involved increases in striatal activity. Some differences pointing to possible WM-specific activation increases in the vicinity of the premotor cortex could not be confirmed with a direct contrast, as the number of studies was too small. The reported results related to automatic vs. controlled processing could hence be interpreted mainly as training-induced changes in task-specific utilization of shared attention control systems and PM representations. One novel finding, however, is that a similar prefrontal-increase parietal-decrease pattern that has been suggested to predict performance in cross-sectional WM studies (Braver et al. 1997, Honey et al. 2000, see also Pessoa et al. 2002, Linden et al. 2003, Olesen et al. 2004) is also observed in PM learning.

Early studies on sensory-motor and language learning relying on spatially less accurate PET findings and very small sample sizes reported widespread activation decreases, interpreted as increased automaticity, and clearly less activation increases (Chein and Schneider 2005). Later 
studies included in the meta-analyses by Davis and Gaskell 2009 and Hardwick et al. 2013 suggest a different pattern. Activation decreases consistent across all studies were only observed in DLPFC, but when separating spatial, motor and verbal tasks, more decreases were found for the visual and motor areas (Figure 6). Based on the present findings, PM training effects should not be characterized as an across-the-board automatization but rather a shift in balance, with up-regulation of domain-general attention networks and down-regulation of perceptual representations and higher level controlled processing (see Chein and Schneider 2012). WM training effects, in turn, were not only observed as enhanced activity in the fronto-parietal networks, but also as decreased activity in areas involved in perceptual processing. In both instances, the activation decreases could be related to more efficient use of perceptual information during the delay periods (Luck \& Vogel 2013). Hence, our results provide converging evidence that puts WM and PM training studies into a more general context of domain-general learning. Even though no direct conclusions about the possible brain plasticity resulting from WM and PM training can be made based on these results, these findings give no reason to assume that the neurobiological mechanisms of these two types of behavioral interventions would differ. In both cases, it is likely that the loci of modulated activity are strongly determined by the areas directly involved in the trained task.

\section{Which of the observed brain activation changes following WM training reflect transfer to}

untrained tasks? Since a multitude of goal-directed functions rely on WM, the question of trainingrelated transfer is crucial for the relevance of WM training. Without behavioral transfer, it is difficult to draw any strong conclusions of the neural effects of WM training on other than the trained task. Transfer effects in published brain imaging studies mainly concern structurally similar tasks. Even though all published studies examining near transfer effects (see Table 1) have reported some training-related activation changes, the data base is still meager for a meta-analysis.

Our results provided evidence for the role of the fronto-striatal system in the mediation of WM 
transfer effects (Figure 7, for a review see Bäckman and Nyberg 2013). In addition to these fMRI findings, also a recent PET-dopamine study reported transfer-related effects in these regions (Bäckman et al. 2017). Moreover, a similar DLPFC activation decrease vs. striatal activation increase pattern, as indicated by the present findings, has been reported previously (Dahlin et al. 2008). As described above, the frontostriatal system plays a key role in linking cognitive functions with the motor system. Thus, in accordance with the recent meta-analyses of behavioral data (Melby-Lervåg and Hulme 2016, Soveri et al. 2017), the functional roles of the transfer-related regions showing enhanced brain activity suggest that near transfer may not be based on modulation of core WM processes, but on the development of relatively task-specific skills. Our preliminary findings related to separate analyses for studies with short or long training period suggested that the modulations in the fronto-striatal circuitries may require some time to emerge (Figure 5). As the number of studies is not yet quite sufficient for a direct contrast between short vs. long training, and as especially PM studies show modulations of fronto-striatal circuitries already after short training, these results should be interpreted with caution. Also the number of foci for transfer-related activation increases and decreases is still very limited for meta-analytical purposes.

The existing brain imaging studies are not well suited in addressing the important question whether WM training yields transfer beyond the WM domain, a question that has been highlighted in behavioral meta-analyses. The main reason for this is that in fMRI studies the focus has been so clearly in near transfer. Moreover, a comprehensive analysis of far-transfer effects with ALE approach might be problematic since different types of far-transfer measures could involve distinct brain networks and such findings are difficult to account for in a regional meta-analysis. Since the current behavioral transfer findings in WM training studies are not very promising, at the moment this may not be a path worth following. Rather, a closer look at the neural mechanisms of the wellestablished task-specific near transfer effects in WM training and the nature of considerable interindividual differences in training outcomes could be worth undertaking. 
Main limitations of the existing data. One of the key challenges for meta-analyses of both behavioral and brain imaging data on WM training stems from the heterogeneity of the available data in terms of training regimes, pre- and posttests used, learning effects (e.g., changes in accuracy or reaction speed), and load effects (e.g, difference in task load between the training period and preposttest, see Supplementary Table 2). Even though the meta-analytical approach can be expected to reveal the most robust effects, there could be valuable information underlying these diverse mediators of training. Due to the distinct effects of reaction time and accuracy on brain activity, relative changes in these measures could potentially explain some variability in fMRI signal, for instance, one resulting to differential activation increase vs. decrease patterns. It should be noted, however, that based on the existing findings, it is likely that at least changes in response speed as such occur outside the core WM systems (Takeuchi et al. 2011). Nevertheless, task demands and learning effects would optimally be accounted for in the analysis of the training-related brain imaging data. Available data does not yet allow to establish such analysis with the means of ALE meta-analysis, but this issue could be further examined when the amount of data accumulates. Another factor that is potentially causing heterogeneity in the reported results is the type of stimulus materials used. We conducted separate analyses for verbal and spatial training effects to specifically examine the role of stimulus materials (verbal vs. visuospatial) in training effects. Our preliminary analysis (see Figure 4, Supplementary Figure 1) suggested that for the verbal tasks, consistent activation changes are reported in the dorsal attention network and salience network (including the Broca's area). In the spatial WM tasks, in turn, the most robust effects are reported in the right DLPFC. These results are consistent with the existing knowledge on the role of specialization to verbal and spatial representations (see also Figures 2 and 3). Probably due to too small samples, especially spatial WM training effects were limited to more confined brain areas than would be expected. Indeed, activation changes in this even more heterogenous and small sample have been reported in widespread brain networks, but due to variability of the activated areas, statistically 
significant effects were observed only in limited part of this network (see Supplementary Figure 1). Hence, with the current limited set of available studies, the effects of task paradigm or stimulation type are not yet reliably discernible and the relatively small body of studies hampers the use of stringent statistical thresholding in such analysis.

Conclusion. So far, brain imaging studies have provided evidence of multiple distinct influences of WM training on brain function. For instance, WM training significantly improves decoding of taskrelevant stimulus information (Meyers et al. 2012), enhances brain connectivity (Jolles et al. 2013, Astle et al. 2015), triggers a neuromodulatory response (McNab et al. 2009, Bäckman et al. 2011), and is dependent on specific gene regulation (Söderqvist et al. 2014). However, four key questions have remained unanswered. (1) Are activation changes induced by WM training observed only within the canonical WM network (redistribution of WM)? The present response is yes, as we observed no allocation of new brain areas that would speak for reorganization of WM-related networks. (2) Are the activation changes related to WM training similar to those reported in learning of PM tasks? Again, the response is yes: our results are mostly in agreement with the suggested domain-general brain network for learning (Chein and Schneider 2012), with only DLPFC/VLPFC showing more consistent training-related modulations specifically for WM training, likely due to their well-established role in short-term maintenance and rehearsal. (3) Which brain networks manifest changes in automatic vs. controlled processes in WM vs. PM training? Both types of training yielded similar, bidirectional dynamics with increased automatization related to more efficient use of perceptual representations, and enhanced controlled processing in the frontoparietal networks. This further suggests that WM is placed on a more general perceptionaction cycle (see Nyberg and Eriksson 2015). (4) Which of the observed brain activation changes following WM training reflect transfer to untrained tasks? The available fMRI data is rather limited to answer to this question, and the only region we could provisionally link to near transfer was the fronto-striatal system. The lack of other mechanisms than modulation of the system mostly related 
to skill learning could be related to the very limited behavioral transfer seen in recent metaanalyses.

\section{Acknowledgements}

ML was supported by the Åbo Akademi University Endowment (the BrainTrain project) and by the Academy of Finland (grant \#260276). Special thanks to all members of the BrainTrain research group and Professor Lars Bäckman for their valuable comments.

\section{References}

Alexander, G.E., DeLong, M.R., Strick, P.L., 1986. Parallel organization of functionally segregated circuits linking basal ganglia and cortex. Annu. Rev. Neurosci. 9, 357-381.

Astle, D.E., Barnes, J.J., Baker, K., Colclough, G.L., Woolrich, M.W., 2015. Cognitive training enhances intrinsic brain connectivity in childhood. J. Neurosci. 35, 6277-6283.

Au, J., Sheehan, E., Tsai, N., Duncan, G.J., Buschkuehl, M., Jaeggi, S.M., 2015. Improving fluid intelligence with training on working memory: a meta-analysis. Psychon Bull Rev. 22, 366-377.

Bäckman, L., Nyberg, L., 2013. Dopamine and training-related working-memory improvement. Neurosci. Biobehav. Rev. 37, 2209-2219.

Bäckman, L., Nyberg, L., Soveri, A., Johansson, J., Andersson, M., Dahlin, E., Neely, A.S., Virta, J., Laine, M., Rinne, J.O., 2011. Effects of working-memory training on striatal dopamine release. Science 333, 718.

Bäckman, L., Waris, O., Johansson, J., Andersson, M., Rinne, J.O., Alakurtti, K., Soveri, A., Laine, M., Nyberg, L., 2017. Increased dopamine release after working-memory updating training: Neurochemical correlates of transfer. Sci. Rep. 7, 7160-017-07577-y. 
Baddeley, A. D. (1986). Working memory. Oxford: Oxford University Press.

Badre, D., Wagner, A.D., 2007. Left ventrolateral prefrontal cortex and the cognitive control of memory. Neuropsychologia 45, 2883-2901.

Barbey, A.K., Koenigs, M., Grafman, J., 2013. Dorsolateral prefrontal contributions to human working memory. Cortex 49, 1195-1205.

Beatty, E.L., Jobidon, M.E., Bouak, F., Nakashima, A., Smith, I., Lam, Q., Blackler, K., Cheung, B., Vartanian, O., 2015. Transfer of training from one working memory task to another: behavioural and neural evidence. Front Syst Neurosci. 9, 86.

Bergman-Nutley, S., Klingberg, T., 2014. Effect of working memory training on working memory, arithmetic and following instructions. Psychol Res. 78, 869-77.

Binder, J.C., Martin, M., Zöllig, J., Röcke, C., Mérillat, S., Eschen, A., Jäncke, L., Shing, Y.L., Multi-domain training enhances attentional control. Psychol Aging. 31, 390-408.

Blumenfeld, R.S., Ranganath, C., 2007. Prefrontal cortex and long-term memory encoding: an integrative review of findings from neuropsychology and neuroimaging. Neuroscientist 13, 280291.

Boyden, E.S., Katoh, A., Raymond, J.L., 2004. Cerebellum-dependent learning: the role of multiple plasticity mechanisms. Annu. Rev. Neurosci. 27, 581-609.

Braver, T.S., Barch, D.M., Kelley, W.M., Buckner, R.L., Cohen, N.J., Miezin, F.M., Snyder, A.Z., Ollinger, J.M., Akbudak, E., Conturo, T.E., Petersen, S.E., 2001. Direct comparison of prefrontal cortex regions engaged by working and long-term memory tasks. Neuroimage 14, 48-59.

Braver, T.S., Cohen, J.D., Nystrom, L.E., Jonides, J., Smith, E.E., Noll, D.C., 1997. A parametric study of prefrontal cortex involvement in human working memory. Neuroimage 5, 49-62.

Brehmer, Y., Westerberg, H., Bäckman, L., 2012. Working-memory training in younger and older adults: training gains, transfer, and maintenance. Front Hum Neurosci. 27, 63. 
Buonomano, D.V., Merzenich, M.M., 1998. Cortical plasticity: from synapses to maps. Annu Rev Neurosci. 21, 149-86.

Buschkuehl, M., Jaeggi, S.M., Jonides, J., 2012. Neuronal effects following working memory training. Dev. Cogn. Neurosci. 2 Suppl 1, S167-79.

Cabeza, R., Dolcos, F., Graham, R., Nyberg, L., 2002. Similarities and differences in the neural correlates of episodic memory retrieval and working memory. Neuroimage 16, 317-330.

Chein, J.M., Morrison, A.B., 2010. Expanding the mind's workspace: training and transfer effects with a complex working memory span task. Psychon Bull Rev. 17, 193-1999.

Chein, J.M., Schneider, W., 2005. Neuroimaging studies of practice-related change: fMRI and meta-analytic evidence of a domain-general control network for learning. Brain Res. Cogn. Brain Res. 25, 607-623.

Chein, J.M., Schneider, W., 2012. The brain's learning and control architecture. Curr. Dir. Psychol. Sci. $21,78-84$.

Cheng, Y., Wu, W., Feng, W., Wang, J., Chen, Y., Shen, Y., Li, Q., Zhang, X., Li, C., 2012. The effects of multi-domain versus single-domain cognitive training in non-demented older people: a randomized controlled trial. BMC Med. 27, 10-30.

Choi, E.Y., Tanimura, Y., Vage, P.R., Yates, E.H., Haber, S.N., 2017. Convergence of prefrontal and parietal anatomical projections in a connectional hub in the striatum. NeuroImage 146, 821-832 Cole, M.W., Bagic, A., Kass, R., Schneider, W., 2010. Prefrontal dynamics underlying rapid instructed task learning reverse with practice. J. Neurosci. 30, 14245-14254.

Constantinidis, C., Klingberg, T., 2016. The neuroscience of working memory capacity and training. Nat. Rev. Neurosci. 17, 438-449.

Corbetta, M., Shulman, G.L., 2002. Control of goal-directed and stimulus-driven attention in the brain. Nat. Rev. Neurosci. 3, 201-215. 
Crottaz-Herbette, S., Anagnoson, R.T., Menon, V., 2004. Modality effects in verbal working memory: differential prefrontal and parietal responses to auditory and visual stimuli. Neuroimage $21,340-351$.

Culham, J.C., Valyear, K.F., 2006. Human parietal cortex in action. Curr. Opin. Neurobiol. 16, 205212.

Curtis, C.E., D'Esposito, M., 2003. Persistent activity in the prefrontal cortex during working memory. Trends Cogn. Sci. 7, 415-423.

Dahlin, E., Neely, A.S., Larsson, A., Bäckman, L., Nyberg, L., 2008. Transfer of learning after updating training mediated by the striatum. Science 320, 1510-1512.

Davis, M.H., Gaskell, M.G., 2009. A complementary systems account of word learning: neural and behavioural evidence. Philos. Trans. R. Soc. Lond. B. Biol. Sci. 364, 3773-3800.

Duncan, J., 2010. The multiple-demand (MD) system of the primate brain: mental programs for intelligent behaviour. Trends Cogn. Sci. 14, 172-179.

Eickhoff, S.B., Laird, A.R., Grefkes, C., Wang, L.E., Zilles, K., Fox, P.T., 2009. Coordinate-based activation likelihood estimation meta-analysis of neuroimaging data: a random-effects approach based on empirical estimates of spatial uncertainty. Hum. Brain Mapp. 30, 2907-2926.

Eriksson, J., Vogel, E.K., Lansner, A., Bergstrom, F., Nyberg, L., 2015. Neurocognitive Architecture of Working Memory. Neuron 88, 33-46.

Garner, K.G., Dux, P.E., 2015.

Training conquers multitasking costs by dividing task representations in the frontoparietalsubcortical system. Proc Natl Acad Sci U S A. 17, 14372-14377.

Goldman-Rakic, P.S., 1996. Regional and cellular fractionation of working memory. Proc. Natl. Acad. Sci. U. S. A. 93, 13473-13480. 
Goodale, M.A., Milner, A.D., 1992. Separate visual pathways for perception and action. Trends Neurosci. 15, 20-25.

Goulden, N., Khusnulina, A., Davis, N.J., Bracewell, R.M., Bokde, A.L., McNulty, J.P., Mullins, P.G., 2014. The salience network is responsible for switching between the default mode network and the central executive network: replication from DCM. Neuroimage 99, 180-190.

Hardwick, R.M., Rottschy, C., Miall, R.C., Eickhoff, S.B., 2013. A quantitative meta-analysis and review of motor learning in the human brain. Neuroimage 67, 283-297.

Henson, R.N., Rugg, M.D., Shallice, T., Josephs, O., Dolan, R.J., 1999. Recollection and familiarity in recognition memory: an event-related functional magnetic resonance imaging study. J. Neurosci. 19, 3962-3972.

Hickok, G., Poeppel, D., 2004. Dorsal and ventral streams: a framework for understanding aspects of the functional anatomy of language. Cognition 92, 67-99.

Holmes, J., Gathercole, S.E., Dunning, D.L., 2009. Adaptive training leads to sustained enhancement of poor working memory in children. Dev Sci. 12, F9-15.

Holtmaat, A., Svoboda, K., 2009. Experience-dependent structural synaptic plasticity in the mammalian brain. Nat. Rev. Neurosci. 10, 647-658.

Honey, G.D., Bullmore, E.T., Sharma, T., 2000. Prolonged reaction time to a verbal working memory task predicts increased power of posterior parietal cortical activation. Neuroimage 12, 495503.

Hsu, N.S., Novick, J.M., Jaeggi, S.M., 2014. The development and malleability of executive control abilities. Front. Behav. Neurosci. 8, 221.

Jolles, D.D., van Buchem, M.A., Crone, E.A., Rombouts, S.A., 2013. Functional brain connectivity at rest changes after working memory training. Hum. Brain Mapp. 34, 396-406. 
Kane, M.J., Conway, A.R., Miura, T.K., Colflesh, G.J., 2007. Working memory, attention control, and the N-back task: a question of construct validity. J. Exp. Psychol. Learn. Mem. Cogn. 33, 615622.

Kane, M.J., Engle, R.W., 2002. The role of prefrontal cortex in working-memory capacity, executive attention, and general fluid intelligence: an individual-differences perspective. Psychon. Bull. Rev. 9, 637-671.

Karbach, J., Verhaeghen, P., 2014. Making working memory work: a meta-analysis of executivecontrol and working memory training in older adults. Psychol Sci. 25, 2027-2037.

Karr, J.E., Areshenkoff, C.N., Rast, P., Garcia-Barrera, M. A., 2014. An empirical comparison of the therapeutic benefits of physical exercise and cognitive training on the executive functions of older adults: A meta-analysis of controlled trials. Neuropsychology. 28, 829-845.

Kelly, C., Foxe, J.J., Garavan, H., 2006. Patterns of normal human brain plasticity after practice and their implications for neurorehabilitation. Arch. Phys. Med. Rehabil. 87, S20-9.

Kelly, M.E., Loughrey, D., Lawlor, B.A., Robertson, I.H., Walsh, C., Brennan, S., 2014. The impact of cognitive training and mental stimulation on cognitive and everyday functioning of healthy older adults: a systematic review and meta-analysis. Ageing Res Rev. 15, 28-43.

Klingberg, T., 2010. Training and plasticity of working memory. Trends Cogn. Sci. 14, 317-324.

de Lange, A.G., Bråthen, A.C.S., Rohani, D.A., Grydeland, H., Fjell, A.M., Walhovd, K.B., 2017. The effects of memory training on behavioral and microstructural plasticity in young and older adults. Hum Brain Mapp. 38, 5666-5680.

Levy, R., Goldman-Rakic, P.S., 2000. Segregation of working memory functions within the dorsolateral prefrontal cortex. Exp. Brain Res. 133, 23-32. 
Li, B., Zhu, X., Hou, J., Chen, T., Wang, P., Li, J., 2016.

Combined cognitive training vs. memory strategy training in healthy older adults Front Psychol. 7: 834.

Liakakis, G., Nickel, J., Seitz, R.J., 2011. Diversity of the inferior frontal gyrus--a meta-analysis of neuroimaging studies. Behav. Brain Res. 225, 341-347.

Lilienthal, L., Tamez, E., Shelton, J.T., Myerson, J., Hale, S., 2013. Dual n-back training increases the capacity of the focus of attention. Psychos Bull Rev. 20, 135-41.

Liljeholm, M., O'Doherty, J.P., 2012. Contributions of the striatum to learning, motivation, and performance: an associative account. Trends Cogn. Sci. 16, 467-475.

Linden, D.E., Bittner, R.A., Muckli, L., Waltz, J.A., Kriegeskorte, N., Goebel, R., Singer, W., Munk, M.H., 2003. Cortical capacity constraints for visual working memory: dissociation of fMRI load effects in a fronto-parietal network. Neuroimage 20, 1518-1530.

Luck, S.J., Vogel, E.K., 2013. Visual working memory capacity: from psychophysics and neurobiology to individual differences. Trends Cogn. Sci. 17, 391-400.

Lövdén, M., Bäckman, L., Lindenberger, U., Schaefer, S., Schmiedek, F., 2010. A theoretical framework for the study of adult cognitive plasticity. Psychol Bull. 136, 659-676.

Manelis, A., Reder, L.M., 2014. Effective connectivity among the working memory regions during preparation for and during performance of the n-back task. Front. Hum. Neurosci. 8, 593.

Marvel, C.L., Desmond, J.E., 2010. Functional topography of the cerebellum in verbal working memory. Neuropsychol. Rev. 20, 271-279.

McNab, F., Varrone, A., Farde, L., Jucaite, A., Bystritsky, P., Forssberg, H., Klingberg, T., 2009. Changes in cortical dopamine D1 receptor binding associated with cognitive training. Science 323, 800-802. 
Melby-Lervåg, M., Hulme, C., 2013. Is working memory training effective? A meta-analytic review. Dev Psychol. 49, 270-291.

Melby-Lervag, M., Redick, T.S., Hulme, C., 2016. Working Memory Training Does Not Improve Performance on Measures of Intelligence or Other Measures of "Far Transfer": Evidence From a Meta-Analytic Review. Perspect. Psychol. Sci. 11, 512-534.

Menon, V., Uddin, L.Q., 2010. Saliency, switching, attention and control: a network model of insula function. Brain Struct. Funct. 214, 655-667.

Mesulam, M.M., 1981. A cortical network for directed attention and unilateral neglect. Ann. Neurol. 10, 309-325.

Meyer, T., Qi, X.L., Stanford, T.R., Constantinidis, C., 2011. Stimulus selectivity in dorsal and ventral prefrontal cortex after training in working memory tasks. J Neurosci. 31, 6266-6276.

Meyers, E.M., Qi, X.L., Constantinidis, C., 2012. Incorporation of new information into prefrontal cortical activity after learning working memory tasks. Proc. Natl. Acad. Sci. U. S. A. 109, 46514656.

Middleton, F.A., Strick, P.L., 2000. Basal ganglia and cerebellar loops: motor and cognitive circuits. Brain Res. Brain Res. Rev. 31, 236-250.

Miller, E.K., Cohen, J.D., 2001. An integrative theory of prefrontal cortex function. Annu. Rev. Neurosci. 24, 167-202.

Muller, N.G., Knight, R.T., 2006. The functional neuroanatomy of working memory: contributions of human brain lesion studies. Neuroscience 139, 51-58.

Nee, D.E., Jonides, J., 2008. Neural correlates of access to short-term memory. Proc. Natl. Acad. Sci. U. S. A. 105, 14228-14233. 
Nemmi, F., Helander, E., Helenius, O., Almeida, R., Hassler, M., Räsänen, P., Klingberg, T., 2016. Behavior and neuroimaging at baseline predict individual response to combined mathematical and working memory training in children. Dev Cogn Neurosci. 20, 43-51.

Nilsson, J., Lebedev, A.V., Rydström, A., Lövdén, M., 2017. Direct-current stimulation does little to improve the outcome of working memory training in older adults. Psychol Sci. 28, 907-920.

Nyberg, L., Eriksson, J., 2015. Working Memory: Maintenance, Updating, and the Realization of Intentions. Cold Spring Harb Perspect. Biol. 8, a021816.

Olesen, P.J., Westerberg, H., Klingberg, T., 2004. Increased prefrontal and parietal activity after training of working memory. Nat. Neurosci. 7, 75-79.

Owen, A.M., McMillan, K.M., Laird, A.R., Bullmore, E., 2005. N-back working memory paradigm: a meta-analysis of normative functional neuroimaging studies. Hum. Brain Mapp. 25, 4659.

Passolunghi, M.C., Vercelloni, B., Schadee, H., 2007. The precursors of mathematics learning: Working memory, phonological ability and numerical competence. Cogn. Develop. 22, 165-184.

Pauli, W.M., O'Reilly, R.C., Yarkoni, T., Wager, T.D., 2016. Regional specialization within the human striatum for diverse psychological functions. Proc Natl Acad Sci U S A. 113, 1907-1912.

Pessoa, L., Gutierrez, E., Bandettini, P., Ungerleider, L., 2002. Neural correlates of visual working memory: fMRI amplitude predicts task performance. Neuron 35, 975-987.

Qi, X.L., Meyer, T., Stanford, T.R., Constantinidis, C., 2011. Changes in prefrontal neuronal activity after learning to perform a spatial working memory task. Cereb Cortex. 21, 2722-2732. Rainer, G., Miller, E.K., 2000., Effects of visual experience on the representation of objects in the prefrontal cortex. Neuron 27, 179-189. 
Richmond, L.L., Wolk, D., Chein, J., Olson, I.R., 2014. Transcranial direct current stimulation enhances verbal working memory training performance over time and near transfer outcomes. J Cogn Neurosci. 26, 2443-2454.

Rizzolatti, G., Fogassi, L., Gallese, V., 2002. Motor and cognitive functions of the ventral premotor cortex. Curr. Opin. Neurobiol. 12, 149-154.

Rottschy, C., Langner, R., Dogan, I., Reetz, K., Laird, A.R., Schulz, J.B., Fox, P.T., Eickhoff, S.B., 2012. Modelling neural correlates of working memory: a coordinate-based meta-analysis.

Neuroimage 60, 830-846.

Ruf, S.P., Fallgatter, A.J., Plewnia, C., 2017. Augmentation of working memory training by transcranial direct current stimulation (tDCS). Sci Rep. 21, 876.

Rugg, M.D., Vilberg, K.L., 2013. Brain networks underlying episodic memory retrieval. Curr. Opin. Neurobiol. 23, 255-260.

Sakai, K., Kitaguchi, K., Hikosaka, O., 2003. Chunking during human visuomotor sequence learning. Exp. Brain Res. 152, 229-242.

Salmi, J., Glerean, E., Jääskeläinen, I.P., Lahnakoski, J.M., Kettunen, J., Lampinen, J., Tikka, P., Sams, M., 2014. Posterior parietal cortex activity reflects the significance of others' actions during natural viewing. Hum. Brain Mapp. 35, 4767-4776.

Salmi, J., Pallesen, K.J., Neuvonen, T., Brattico, E., Korvenoja, A., Salonen, O., Carlson, S., 2010. Cognitive and motor loops of the human cerebro-cerebellar system. J. Cogn. Neurosci. 22, 26632676.

Salminen, T., Mårtensson, J., Schubert, T., Kühn, S., 2016. Increased integrity of white matter pathways after dual n-back training.Neuroimage. 133, 244-250. 
Saur, D., Kreher, B.W., Schnell, S., Kummerer, D., Kellmeyer, P., Vry, M.S., Umarova, R., Musso, M., Glauche, V., Abel, S., Huber, W., Rijntjes, M., Hennig, J., Weiller, C., 2008. Ventral and dorsal pathways for language. Proc. Natl. Acad. Sci. U. S. A. 105, 18035-18040.

Schneider, W., Shiffrin, R. M., 1977. Controlled and automatic human information processing: I. Detection, search, and attention. Psychol. Rev. 84, 1-66.

Schmiedek, F., Lovden, M., Lindenberger, U., 2014. A task is a task is a task: putting complex span, n-back, and other working memory indicators in psychometric context. Front. Psychol. 5, 1475.

Schneider-Garces, N.J., Gordon, B.A., Brumback-Peltz, C.R., Shin, E., Lee, Y., Sutton, B.P., Maclin, E.L., Gratton, G., Fabiani, M., 2010. Span, CRUNCH, and beyond: working memory capacity and the aging brain. J Cogn Neurosci. 22, 655-669.

Scimeca, J.M., Badre, D., 2012. Striatal contributions to declarative memory retrieval. Neuron 75, 380-392.

Scolari, M., Seidl-Rathkopf, K.N., Kastner, S., 2015. Functions of the human frontoparietal attention network: Evidence from neuroimaging. Curr. Opin. Behav. Sci. 1, 32-39.

Shinaver, C.S., Entwistle, P.C., Söderqvist, S., 2014. Cogmed WM training: reviewing the reviews. Appl Neuropsychol Child. 3, 163-72.

Seidler, R.D., Bo, J., Anguera, J.A., 2012. Neurocognitive contributions to motor skill learning: the role of working memory. J. Mot. Behav. 44, 445-453.

Silver, M.A., Kastner, S., 2009. Topographic maps in human frontal and parietal cortex. Trends Cogn. Sci. 13, 488-495.

Smith, E.E., Jonides, J., 1999. Storage and executive processes in the frontal lobes. Science 283, $1657-1661$. 
Söderqvist, S., Matsson, H., Peyrard-Janvid, M., Kere, J., Klingberg, T., 2014. Polymorphisms in the dopamine receptor 2 gene region influence improvements during working memory training in children and adolescents. J. Cogn. Neurosci. 26, 54-62.

Soveri, A., Antfolk, J., Karlsson, L., Salo, B., Laine, M., 2017. Working memory training revisited: A multi-level meta-analysis of n-back training studies. Psychon. Bull. Rev. .

St Clair- Thompson, H., Stevens, R., Hunt, A., Bolder, E., 2010. Improving children's working memory and classroom performance Edu Psychol. 203-219.

Stephens, J.A., Jones, K.T., Berryhill, M.E., 2017. Task demands, tDCS intensity, and the COMT val158met polymorphism impact tDCS-linkedworking memory training gains. Sci Rep. 18, 13463.

Takeuchi, H., Sekiguchi, A., Taki, Y., Yokoyama, S., Yomogida, Y., Komuro, N., Yamanouchi, T., Suzuki, S., Kawashima, R., 2010. Training of working memory impacts structural connectivity. J. Neurosci. 30, 3297-3303.

Takeuchi, H., Taki, Y., Nouchi, R., Hashizume, H., Sekiguchi, A., Kotozaki, Y., Nakagawa, S., Miyauchi, C.M., Sassa, Y., Kawashima, R., 2013. Effects of working memory training on functional connectivity and cerebral blood flow during rest. Cortex 49, 2106-2125.

Thorell, L.B., Lindqvist, S., Bergman-Nutley, S., Bohlin, G., Klingberg, T. 2009. Training and transfer effects of executive functions in preschool children. Dev Sci. 12, 106-13.

Turkeltaub, P.E., Eickhoff, S.B., Laird, A.R., Fox, M., Wiener, M., Fox, P., 2012. Minimizing within-experiment and within-group effects in Activation Likelihood Estimation meta-analyses. Hum. Brain Mapp. 33, 1-13.

Turley-Ames, K.J., Whitfield, M. M., 2003. Strategy training and working memory task performance. J. Mem. Lang., 49, 446-468

van Polanen, V., Davare, M., 2015. Interactions between dorsal and ventral streams for controlling skilled grasp. Neuropsychologia 79, 186-191. 
von Bastian, C.C., Oberauer, K., 2014. Effects and mechanisms of working memory training: a review. Psychol. Res. 78, 803-820.

Wenger, E., Brozzoli, C., Lindenberger, U., Lövdén, M., 2017. Expansion and Renormalization of Human Brain Structure During Skill Acquisition. Trends Cogn Sci. 21, 930-939.

Zatorre, R.J., Fields, R.D., Johansen-Berg, H.. 2012. Plasticity in gray and white: neuroimaging changes in brain structure during learning. Nat Neurosci. 15, 528-536.

\section{Figure legends}

Figure 1. The general framework for the meta-analyses conducted in the present study. (1) To provide a basis for examining whether the training effects represent reconfiguration of the largescale brain networks (allocation of new brain areas) or redistribution of the task-related brain networks (modulation of the same areas), we collected extensive data from Neurosynth database and conducted term-based meta-analyses. (2) WM training data was collected by utilizing several search engines and from papers included in previous reviews on this topic (see Methods section for details). To distinguish between WM core processes and task-specific representations, we also collected data regarding training of task-specific PM representations from prior meta-analyses on PM learning (Chein and Schneider 2005, Davis and Gaskell 2009, Hardwick et al. 2013). (3) We then conducted ALE meta-analyses across all WM and PM training-related activation changes. In these analyses, we also separated between verbal and spatial WM training studies and WM training studies with short (less than two weeks) or long (more than two weeks) training period. PM training studies were divided to those involving verbal, spatial, or motor tasks. (4) After this, we separately examined whether the patterns of activity decreases and increases, assumed to reflect changes in the balance between automatic and controlled processing, are similar across WM and PM studies. (5) Finally, we separated between training and transfer effects reported in WM studies. 
Figure 2. a) Illustration of the key brain networks previously considered to be involved in WM. b) WM- and PM-related brain areas that are activated across large-scale datasets obtained from NeuroSynth (www.neurosynth.org). Colormaps are additive, with white colored areas representing brain regions involved in all WM and PM tasks. c) Areas activated in widespread studies reporting learning effects for perceptual, motor, and memory-related studies (NeuroSynth). The meta-analysis maps are thresholded at FDR corrected $p<0.01$

Figure 3. a) Areas showing consistent WM or PM learning effects are observed in specialized areas of the learning-related network (corrected $p<0.05$ ). b) Contrast analysis (uncorrected $p<0.01$ ) revealed the areas showing significant differences between WM and PM learning networks.

Figure 4. WM training-related activations separately analyzed for studies with verbal or spatial tasks. Both maps are thresholded at corrected $p<0.05$.

Figure 5. WM training-related activations in studies with short $(<2$ weeks $)$ or long training period. Purple color shows the overlapping activations. Both maps are thresholded at corrected $p<0.05$.

Figure 6. a) Increases and decreases of brain activity across all WM and PM training studies. Separate analyses for activation increases/decreases for b) WM-training, and c) verbal learning, d) spatial learning, and e) motor learning. All maps are thresholded at corrected $p<0.05$.

Figure 7. a) Activation changes measured in trained and untrained WM tasks. Overlap between the consistent activations in the trained and untrained tasks is shown in white color. Results of the analyses separating between activation increases and decreases for the trained and untrained tasks 
are illustrated in b) and c), respectively. All maps are thresholded at corrected $p<0.05$.

Figure 8 Illustration of the three subnetworks of WM function and related neurocognitive mechanisms underlying training effects. Down-regulated and up-regulated refer to training-related change towards automatic or controlled processing. 

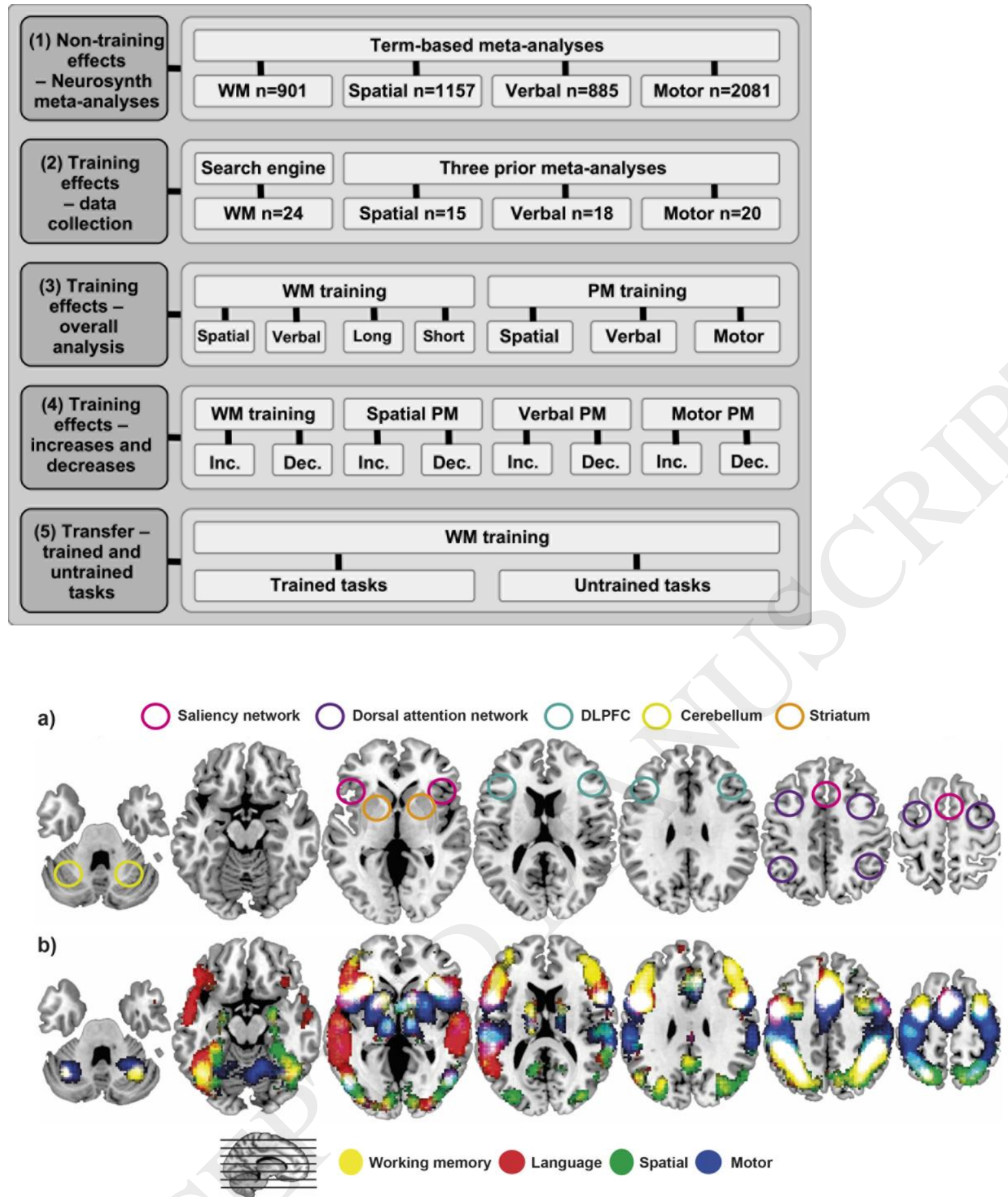


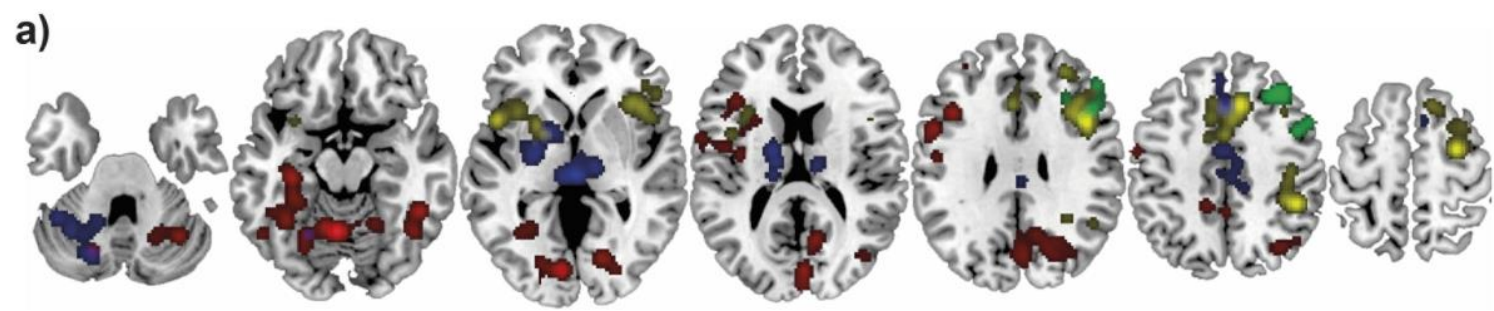

b)

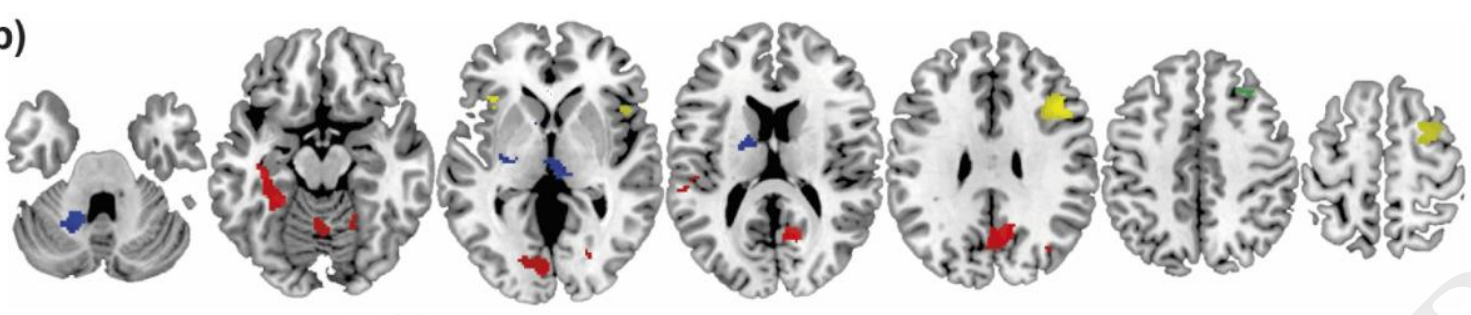

$$
\text { Working memory } \bigcirc \text { Language } \bigcirc \text { Spatial } \bigcirc \text { Motor }
$$

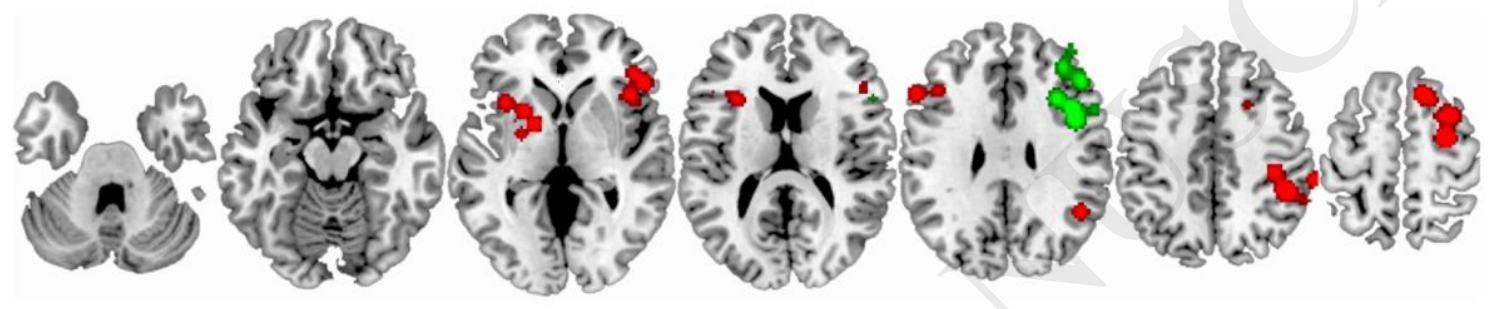

Spatial working memory $\bigcirc$ Verbal working memory

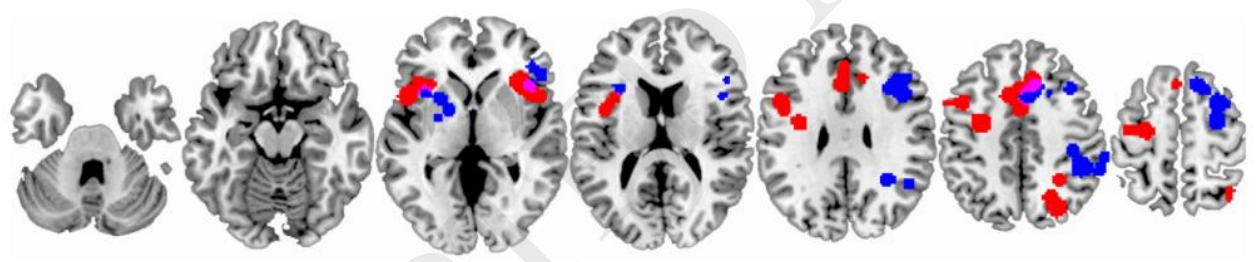

Short training period $\bigcirc$ Long training period 

a)

b)

c)

d)

e)

2.

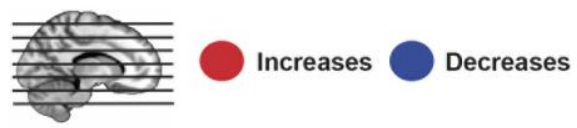


a)
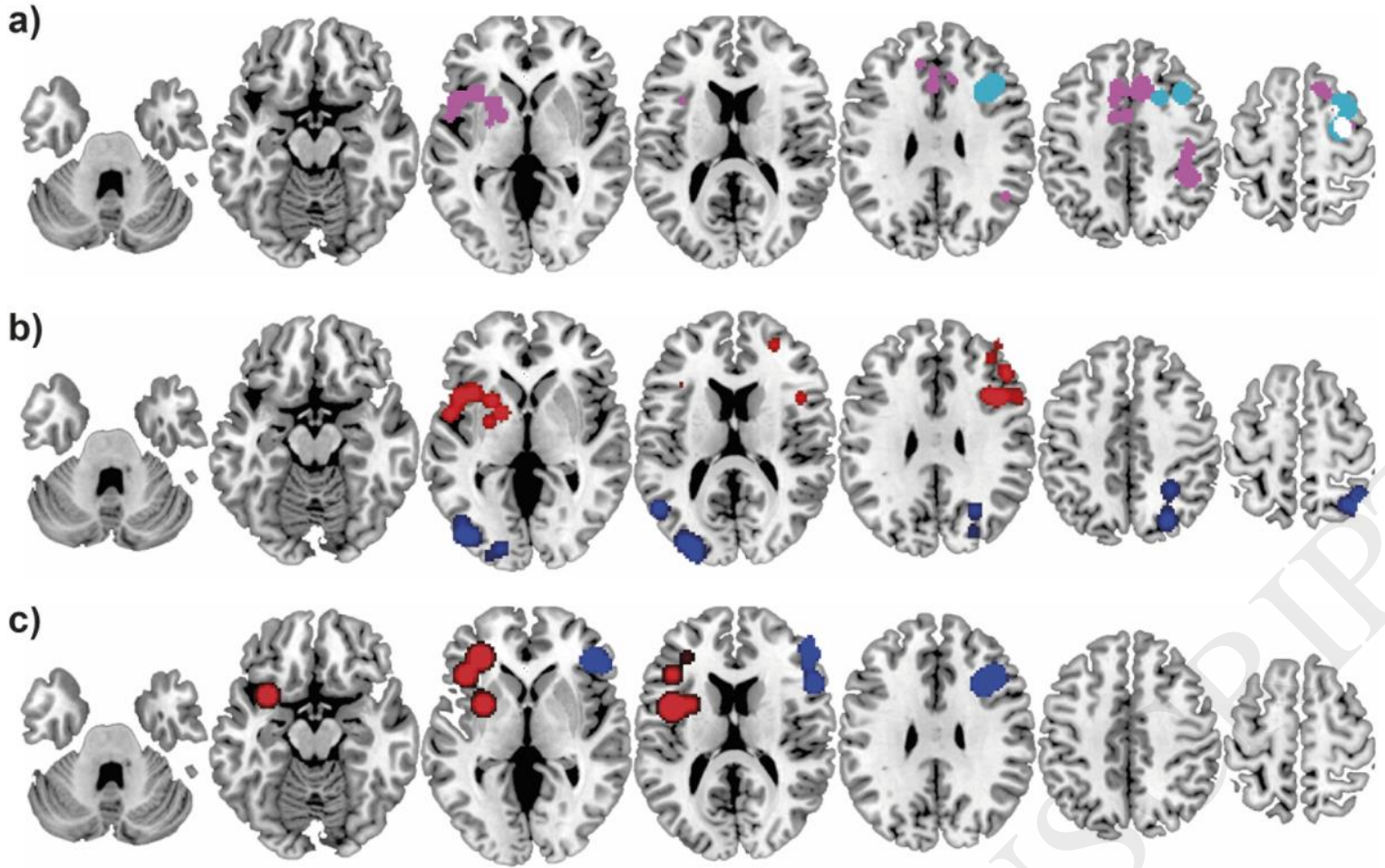

Tranining all $O$ Transfer all $O$ Increases $O$ Decreases

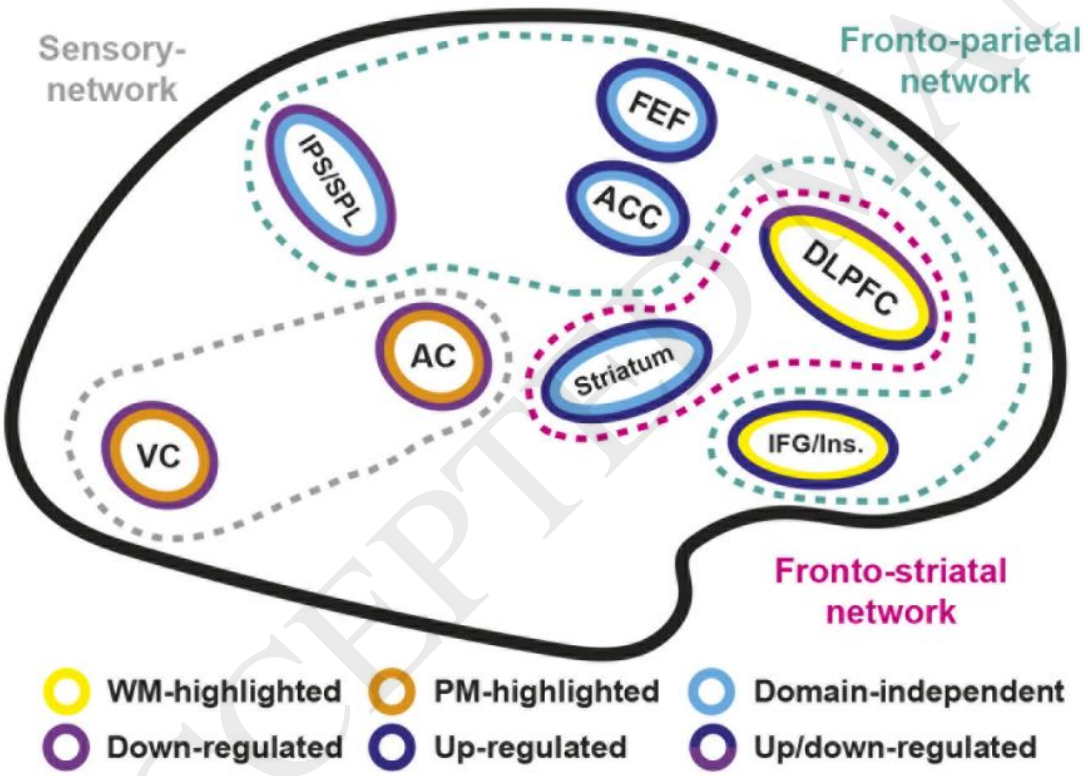


Table 1. Overview of the studies meeting the inclusion criteria for the meta-analysis. $\mathrm{N}$ refers to size of the training group. Transfer effecs are divided to three alternatives: yes $=$ transfer and criterion tasks, no $=$ criterion tasks, only $=$ only transfer effects. In the training period section number of training sessions in paranthesis. dMTS = delayed matching to sample.

\begin{tabular}{|c|c|c|c|c|c|c|}
\hline Study & $\mathrm{N}$ & $\begin{array}{l}\text { Control } \\
\text { group }\end{array}$ & $\begin{array}{l}\text { Transfer } \\
\text { assessed }\end{array}$ & Intervention & $\begin{array}{l}\text { Increase / } \\
\text { decrease }\end{array}$ & $\begin{array}{l}\text { Training } \\
\text { period }\end{array}$ \\
\hline Beatty et al. 2015 & 22 & yes & only & Verbal n-back $\rightarrow$ Visuospatial dMTS & $\uparrow$ & $2 \mathrm{~d}(3)$ \\
\hline Brehmer et al. 2011 & 12 & yes & only & Seven WM tasks $\rightarrow$ Visuospatial dMTS & $\uparrow \downarrow$ & $5 \mathrm{w}(25)$ \\
\hline Buschkuel et al. 2014 & 27 & yes & no & Visuospatial n-back & $\uparrow$ & $7 \mathrm{~d}(7)$ \\
\hline Dahlin et al. 2008 & 12 & yes & yes & Six updating tasks $\rightarrow$ n-back, stroop & $\uparrow \downarrow$ & $5 \mathrm{w}(15)$ \\
\hline Garavan et al. 2000 & 5 & no & no & Visuospatial dMTS & $\uparrow$ & $1 \mathrm{~d}(44)$ \\
\hline Garner \& Dux 2015 & 50 & yes & no & Multitasking & Group $\mathrm{x}$ time & $5 d(5)$ \\
\hline Erickson et al. 2007 & 31 & yes & no & Color-letter dual task & Group $\mathrm{x}$ time & $17 \mathrm{~d}(5)$ \\
\hline Heinzel et al. 2016 & 16 & yes & yes & Number n-back $\rightarrow$ Letter dMTS & Group x time & $4 \mathrm{w}(12)$ \\
\hline Hötting et al. 2013 & 47 & & no & Spatial WM & $\uparrow$ & 6 months \\
\hline Jansma et al. 2001 & 15 & no & no & Verbal dMTS & $\downarrow$ & $1 \mathrm{~d}(4)$ \\
\hline Jolles et al. 2010 & 15 & yes & no & Verbal vs. maintenance and manipulation & Group $\mathrm{x}$ time & $6 \mathrm{w}(18)$ \\
\hline Kirchoff et al. 2012 & 16 & yes & no & Strategy training $\rightarrow$ intentional encoding & $\uparrow$ & $2 \mathrm{w}(2)$ \\
\hline Kuhn et al. 2013 & 26 & yes & no & Spatial n-back, number updating & $\downarrow$ & $50 \mathrm{~d}(54)$ \\
\hline Landau et al. 2004 & 10 & no & no & Facial dMTS & Practise x load & $1 \mathrm{~d}(2)$ \\
\hline Moore et al. 2006 & 11 & yes & no & Four tasks (mostly dMTS) & $\uparrow$ & $10 \mathrm{~d}(7)$ \\
\hline Olesen et al. 2004 & 8 & no & no & Three WM tasks $\rightarrow$ visuospatial & $\uparrow \downarrow$ & $5 \mathrm{w}(25)$ \\
\hline Opitz et al. 2014 & 16 & yes & no & Visuospatial n-back & $\uparrow$ & $2 \mathrm{w}(8-10)$ \\
\hline Salminen et al. 2016 & 18 & yes & yes & Dual n-back task & $\uparrow \downarrow$ & $3 \mathrm{w}(16)$ \\
\hline Sayala et al. 2006 & 10 & no & no & Spatial and object dMTS & Repetition & $1 \mathrm{w}(9)$ \\
\hline Schmicker et al. 2016 & 14 & yes & no & Visuospatial dMTS & $\uparrow \downarrow$ & $5 \mathrm{~d}(5)$ \\
\hline Schneiders et al. 2011 & 48 & yes & yes & Voice, pattern n-back & Group x time & $2 \mathrm{w}(8-10)$ \\
\hline Schneiders et al. 2012 & 16 & yes & only & Voice n-back $\rightarrow$ pattern n-back & Group x time & $3 \mathrm{w}(8)$ \\
\hline Schweizer et al. 2013 & 17 & yes & $\mathrm{O}$ & Emotional dMTS & $\uparrow \downarrow$ & $20 \mathrm{~d}(20)$ \\
\hline Vartanian et al. 2013 & 17 & yes & only & Letter n-back $\rightarrow$ divergent thinking & $\downarrow$ & $3 \mathrm{~d}(3)$ \\
\hline Weissman et al. 2002 & 15 & no & no & Spatial task & $\uparrow \downarrow$ & $1 \mathrm{~d}$ \\
\hline Zimmer et al. 2012 & 12 & no & no & Object dMTS & $\downarrow$ & $4 \mathrm{w}(12)$ \\
\hline
\end{tabular}

